\title{
Riesgo cardiovascular y síndrome metabólico en pacientes VIH positivos en el Caribe colombiano
}

\author{
Cardiovascular risk and metabolic syndrome in \\ HIV-positive patients in the colombian caribbean
}

\author{
Pedro Polo-Acosta, Fernando Romero-Ucrós, Aldo Saumeth-Bovea, \\ Miguel Urina-Triana, Ivan Zuluaga-De LeÓn • Barranguilla \\ Nelson Alvis-Guzmán • Cartagena (Colombia)
}

\section{Resumen}

Objetivo: estimar el riesgo cardiovascular y la prevalencia de síndrome metabólico de un grupo de pacientes VIH positivos de la región caribe colombiana.

Métodos: se llevó a cabo un estudio descriptivo de un grupo de 90 pacientes de ambos géneros, VIH positivos que asisten al programa de control de la EPS SURA. Se estimó la frecuencia síndrome metabólico, riesgo cardiovascular y el tipo de esquema de tratamiento.

Resultados: 72,2\% son hombres con edad media de 39.7 años (IC95\%: 37.3-42.1) la edad promedio general fue de 40.9 años (IC95\%: 38.7-43.0). El 5.4\% eran diabéticos y 14.1\% fueron hipertensos. El $87.8 \%$ recibía algún esquema de tratamiento. La frecuencia de síndrome metabólico fue $46 \%$ en pacientes en tratamiento con inhibidores de proteasa frente a un $36 \%$ en los que no usan tratamiento. Casi la mitad del grupo tenía un índice de masa corporal (IMC) en sobrepeso y obesidad (36 y $13 \%$ respectivamente).

Conclusiones: se detectó un porcentaje alto de insulinorresistencia (36\%) en nuestros pacientes VIH positivos. Se requieren medidas eficaces para prevenir el curso natural de esta entidad. (Acta Med Colomb 2013; 38: xx-xx).

Palabras claves: Virus de la inmunodeficiencia humana, riesgo cardiovascular, síndrome metabólico, terapia antirretroviral de alta efectividad.

\footnotetext{
Abstract

Objective: to estimate cardiovascular risk and the prevalence of metabolic syndrome in a group of HIV-positive patients in the Colombian Caribbean region.

Methods: we conducted a descriptive study of a group of 90 patients of both genders HIV positive seen in the control program of EPS SURA . Frequency of metabolic syndrome, cardiovascular risk and type of treatment schedule, were estimated.

Results: $72.2 \%$ were male with an average age of 39.7 years (95\% CI: 37.3-42.1) overall average age was 40.9 years (95\% CI: $38.7-43.0$ ). $5.4 \%$ had diabetes and $14.1 \%$ were hypertensive. $87.8 \%$ received a treatment regimen. The frequency of metabolic syndrome was $46 \%$ in patients treated with protease inhibitors compared to $36 \%$ in those not being treated. Almost half of the group had a body mass index (BMI) in overweight and obesesity (36 and 13\% respectively).

Conclusions: we found a high percentage of insulin resistance (36\%) in our HIV positive patients. Effective measures are required to prevent the natural course of this entity. (Acta Med Colomb 2013; 38: $\mathrm{xx}-\mathrm{xx})$.

Keywords: human immunodeficiency virus, cardiovascular risk, metabolic syndrome, highly effective antiretroviral therapy.
}

Dres. Pedro Polo-Acosta, Fernando RomeroUcrós, Aldo Saumeth-Bovea, Iván ZuluagaDe León: EPS SURA, Barranquilla; Dr. Miguel Urina-Triana: EPS SURA, Barranquilla, Grupo de investigación Cardiodiagnóstico/ Fundación del Caribe para la Investigación Biomédica. Universidad Simón Bolívar, Barranquilla; Dr. Nelson Alvis-Guzmán: Grupo de Investigación en Economía de la Salud. Universidad de Cartagena (Colombia). Correspondencia. Dr. Pedro Polo-Acosta. E-mail: ppoloacosta@hotmail.com Recibido: 15/X/2012 Aceptado: 25/VI/2013 
La terapia antirretroviral de alta efectividad (TARAE) ha tenido un alto impacto en la reducción de la morbimortalidad asociada a la infección por el virus de la inmunodeficiencia humana (VIH) tipo 1 (1). Sin embargo, desde finales de la década del 90 se han reportado estudios que muestran una relación entre la TARAE y el incremento del riesgo de enfermedad coronaria (2). Esto se deriva de hallazgos de investigaciones como el estudio DAD (Data collection on Adverse events of anti-HIV Drugs) (3), los cuales indican que la incidencia del infarto agudo del miocardio se aumenta con la exposición prolongada a la TARAE (4). La mayoría de los estudios sugieren que la exposición a la TARAE aumenta el riesgo de enfermedad coronaria entre $1.5-2$ veces en los pacientes VIH positivos, al compararlos con controles no infectados pero con bajas tasas de riesgo absoluto (5).

La enfermedad coronaria en los pacientes VIH positivos tiende a aparecer 10 años más tempranamente que en los controles VIH negativos (edad promedio 50 años), afecta más a hombres, se asocia con niveles de colesterol de alta densidad (HDL) disminuido, enfermedad de un solo vaso coronario más que de múltiples vasos y mayor incidencia de complicaciones asociadas al stent intracoronario (6). Las causas de la aterosclerosis asociada al VIH son la dislipidemia, insulinorresistencia, inflamación y cambios en la composición corporal. Estos factores interaccionan con la infección por el $\mathrm{VIH}$, la inflamación asociada a la misma y los efectos adversos de los medicamentos antirretrovirales. Hay una necesidad crítica de detectar, tamizar y tratar adecuadamente la enfermedad cardiovascular en los pacientes VIH positivos (7).

El síndrome metabólico hace referencia a la presencia de múltiples factores de riesgo tales como la obesidad abdominal, aumento de los niveles de la glucosa, elevación de la presión arterial y alteraciones tales como la hipertrigliceridemia y la presencia de un colesterol HDL en niveles bajos. Este síndrome se asocia con un incremento de tres a seis veces del riesgo para presentar diabetes y casos nuevos de hipertensión arterial, además de ser un desencadenante de enfermedad cardiovascular. En Colombia, Lopez (2009) afirma que los criterios diagnósticos de la International Diabetes Federation (IDF) son los más apropiados, para identificar individuos con síndrome metabólico (8). Por otra lado, la lipodistrofia en los pacientes con VIH (evento caracterizado por la pérdida de grasa subcutánea en cara, brazos y piernas en quieres reciben TARAE que incluya inhibidores de la proteasa) puede relacionarse con insulinorresistencia, niveles elevados de triglicéridos y disminuidos de colesterol de alta densidad (10). Algunos pacientes pueden presentar también acumulación de grasa en el cuello y parte superior de la espalda (9).

El presente estudio pretende estimar el riesgo cardiovascular y la prevalencia de síndrome metabólico de un grupo de pacientes VIH positivos de la región caribe colombiana, afiliados a la empresa promotora de salud (EPS) SURA. Además, evaluaremos la presencia o no de lipodistrofia.

\section{Métodos}

El presente es un estudio observacional de corte transversal que busca estimar el riesgo cardiovascular y la prevalencia de síndrome metabólico (SM) de un grupo de pacientes VIH positivos. De igual modo describe los tipos de tratamientos antirretrovirales usados en la población objeto

La población de estudio estuvo compuesta por pacientes VIH positivos que asisten regularmente al programa de control del VIH de la EPS Sura en las ciudades de Barranquilla y Cartagena.

Para la selección de los pacientes por observar se definieron como criterios de inclusión los siguientes: a) pacientes con diagnóstico de infección por VIH, b) de ambos géneros, c) mayores de 18 años, y d) con historia clínica completa. Se excluyeron las pacientes embarazadas.

En todos los pacientes se recolectaron datos relacionados con:

- El esquema de terapia antirretroviral usado. Se determinó el esquema de análogos nucleósidos utilizados como columna vertebral en cada paciente (abacavir más lamivudina, zidovudina más lamivudina, estavudina más lamivudina, didanosina más lamivudina, esquema diferente).

- Los niveles de colesterol de baja densidad, colesterol de alta densidad y triglicéridos.

- El riesgo de desarrollar enfermedad coronaria a 10 años o riesgo cardiovascular global (RCVG) de acuerdo con la escala de Framingham, determinado por el programa de cálculo de riesgo dado por el tercer panel nacional del colesterol en los Estados Unidos (NCEP III), en 2010 (11). Se decidió utilizar la ecuación de riesgo cardiovascular de Framingham, ya que ha demostrado su utilidad en el estudio DAD previamente mencionado, aunque puede subestimar el riesgo en los pacientes VIH positivos fumadores (8). Se usaron las siguientes categorías de riesgo cardiovascular:

Riesgo latente: son las personas que tienen un riesgo menor de $10 \%$ de desarrollar un evento cardiovascular en los próximos 10 años.

Riesgo intermedio: son las personas que tienen un riesgo mayor o igual a $10 \%$ pero menor a $20 \%$ de desarrollar un evento cardiovascular en los próximos 10 años. Aquí también se hallan los pacientes con síndrome metabólico.

Riesgo alto: son las personas que tienen un riesgo mayor o igual a $20 \%$ de desarrollar un evento cardiovascular en los próximos 10 años.

Riesgo muy alto: en esta categoría se incluyen las personas que tienen diabetes mellitus tipo 2, que hayan tenido un evento cardiovascular, o las personas que ya han tenido un evento cardiovascular y tienen dos o más factores de riesgo cardiovascular no controlados. Se denomina evento cardiovascular a: i) enfermedad coronaria previa con o sin síntomas; ii) enfermedad vascular periférica (claudicación intermitente); iii) aneurisma de 
aorta abdominal; iv) enfermedad carotídea aterosclerótica documentada; y v) dislipidemia aterogénica primaria (genética)

- El índice de masa corporal (IMC) de los pacientes

- Presencia o no de lipodistrofia.

- Índice de cintura.

- Tiempo de tratamiento con antirretrovirales.

- El recuento de linfocitos CD4 inicial de cada paciente (al ingreso al programa).

- La presencia o no de SM. Para determinar la presencia de este síndrome se usaron los criterios diagnósticos de la IDF, ya que éstos se han considerado los más adecuados en la población colombiana $(8,12)$. Los criterios para SM fueron:

1. Índice de cintura aumentado: hombres igual o mayor de $90 \mathrm{~cm}$, mujeres igual o mayor de $80 \mathrm{~cm}$. (Parámetro para pacientes de Sudamérica), más dos de los siguientes criterios:

2. Glucosa en ayunas igual o mayor de $100 \mathrm{mg} / \mathrm{dL} \mathrm{o}$ en tratamiento para glucosa elevada.

3. Triglicéridos mayores o iguales a $150 \mathrm{mg} / \mathrm{dL} \mathrm{o}$ paciente en manejo con terapia para triglicéridos elevados (fibratos, acido nicotínico).

4. Colesterol de alta densidad disminuido de acuerdo a sexo (menor de $40 \mathrm{mg} / \mathrm{dL}$ en hombres o $50 \mathrm{mg} / \mathrm{dL}$ en mujeres) o en tratamiento para colesterol de alta densidad disminuido.

5. Presión arterial elevada: tensión arterial sistólica igual o mayor de $130 \mathrm{mmHg}$ y/o diastólica igual o mayor de $85 \mathrm{mmHg}$.

Los datos recolectados fueron ingresados a una base de datos en una hoja de cálculo de Excel $2007^{\mathrm{TM}}$ y analizados usando las herramientas de estadística descriptivas de la misma. Además, para el análisis inferencial se usa el aplicativo EPIDAT 3.1 para análisis epidemiológico de datos tabulados. Para el análisis estadístico de diferencias de medias y contraste de igualdad se utilizó la prueba $t$-Student y se realizó contraste de la igualdad de varianzas basado en la distribución F2.

\section{Resultados}

Se recolectaron los datos clínicos y paraclínicos de 90 pacientes seropositivos para VIH pertenecientes a la Regional Norte de EPS SURA. De éstos 72.2\% son hombres con edad promedio de 39.7 años (IC95\%: 37.3-42.1), la edad promedio de las mujeres fue 43.8 años (IC95\%: 39.2-48.5) y la edad promedio general fue 40.9 años (IC95\%: 38.743.0). El 5.4\% eran diabéticos, $14.1 \%$ hipertensos y sólo $1 \%$ fumaba en el momento del estudio.

El $87.8 \%$ de los pacientes en el momento del estudio recibían TARAE, la mayoría usando esquemas basados en análogos no nucleósidos de la transcriptasa reversa (54.4\%), seguidos de esquemas con inhibidores de la proteasa
(31.1\%). El análogo nucleósido de base más utilizado fue abacavir seguido de la zidovudina.

Al comparar los niveles de triglicéridos en los distintos grupos de tratamiento respecto a los que no recibían tratamiento antirretroviral, se encontró una media de trigliceridemia mayor entre los pacientes que reciben inhibidores de proteasa, siendo esta diferencia estadísticamente significativa $(\mathrm{p}<0.05)$. También se halló una asociación entre la presencia de SM con un recuento de linfocitos CD4 inicial inferior y un índice de masa corporal aumentado.

Comparadas las proporciones de pacientes con y sin SM entre quienes sí y no recibían tratamiento antirretroviral, éstas no fueron estadísticamente diferentes. Un resultado similar se obtuvo al momento de comparar los pacientes agrupados según tipos de antirretrovirales recibidos y no tratamiento.

Al comparar los pacientes agrupados por tipo de esquema antirretroviral, con los que no recibían tratamiento, no se encontraron diferencias significativas en las medias de niveles de colesterol de baja y alta densidad y en el índice de masa corporal.

El riesgo cardiovascular promedio por Framingham fue de $2.3 \%$. El 54\% de los pacientes se clasificaron con riesgo latente y $34 \%$ en riesgo intermedio. La prevalencia de SM fue de $37.8 \%$. La mitad de los pacientes tuvieron sobrepeso u obesidad (36-13\% respectivamente). Más de la mitad de las mujeres $(56.1 \%)$ tuvieron SM comparado con $30 \%$ d los hombres.

En lo referente a tipo de esquema de TARAE usado, los pacientes que recibían inhibidores de proteasa tuvieron mayor porcentaje de criterios para SM (46\%), los de esquema basado en análogos no nucleósidos tuvieron una prevalencia de SM menor a la de los pacientes sin tratamiento antirretroviral (30 y $36 \%$ respectivamente).

El 4.4\% $(n=4)$ presentaron lipodistrofia, siendo más prevalente en mujeres (8\%). La prevalencia de lipodistrofia fue de $7.1 \%$ en pacientes que recibieron inhibidores de proteasa y en los que recibieron un esquema con análogos no nucleósidos la prevalencia fue de $4.1 \%$. Respecto al índice de cintura y al IMC, los pacientes con lipodistrofia no fueron diferentes del resto.

Por otro lado, el índice de cintura no estuvo relacionado con el esquema de tratamiento antirretroviral (Figura 1).

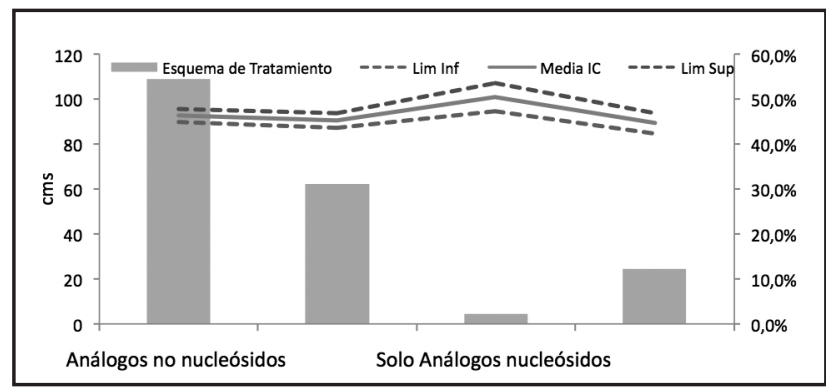

Figura 1. Índice de cintura (IC) y esquema de tratamiento antirretroviral en pacientes con VIH. 
Tabla 1. Distribución de pacientes por edad, género y tipo de tratamiento.

\begin{tabular}{|c|c|c|c|c|c|c|}
\hline & Hombres & $\%$ & Mujeres & $\%$ & Total & $\%$ \\
\hline \multicolumn{7}{|l|}{ Grupo de edad } \\
\hline 21 a 25 & 4 & 4.4 & 0 & 0.0 & 4 & 4.4 \\
\hline 26 a 30 & 10 & 11.1 & 4 & 4.4 & 14 & 15.6 \\
\hline 31 a 35 & 9 & 10.0 & 2 & 2.2 & 11 & 12.2 \\
\hline 36 a 40 & 13 & 14.4 & 4 & 4.4 & 17 & 18.9 \\
\hline 41 a 45 & 13 & 14.4 & 4 & 4.4 & 17 & 18.9 \\
\hline 46 a 50 & 6 & 6.7 & 5 & 5.6 & 11 & 12.2 \\
\hline 51 a 55 & 5 & 5.6 & 3 & 3.3 & 8 & 8.9 \\
\hline 56 a 60 & 5 & 5.6 & 1 & 1.1 & 6 & 6.7 \\
\hline $61 y+$ & 0 & 0.0 & 2 & 2.2 & 2 & 2.2 \\
\hline Total & 65 & 72.2 & 25 & 27.8 & 90 & 100.0 \\
\hline \multicolumn{7}{|c|}{ Tipo de esquema de tratamiento ARV } \\
\hline - Análogos no nucleósidos & 37 & 41.1 & 12 & 13.3 & 49 & 54.4 \\
\hline - Inhibidores de proteasa & 18 & 20.0 & 10 & 11.1 & 28 & 31.1 \\
\hline - Sólo análogos nucleósidos & 1 & 1.1 & 1 & 1.1 & 2 & 2.2 \\
\hline - Sin tratamiento & 9 & 10.0 & 2 & 2.2 & 11 & 12.2 \\
\hline \multicolumn{7}{|l|}{ Síndrome Metabólico } \\
\hline - $\mathrm{Si}$ & 20 & 22.2 & 14 & 15.6 & 34 & 37.8 \\
\hline - No & 45 & 50.0 & 11 & 12.2 & 56 & 62.2 \\
\hline \multicolumn{7}{|l|}{ Diabetes } \\
\hline - $\mathrm{Si}$ & 3 & 3.3 & 2 & 2.2 & 5 & 5.5 \\
\hline • No & 62 & 68.9 & 23 & 25.6 & 85 & 94.5 \\
\hline \multicolumn{7}{|l|}{ Hipertensión arterial } \\
\hline$\cdot \mathrm{Si}$ & 7 & 7.8 & 6 & 6.7 & 13 & 14.4 \\
\hline - No & 58 & 64.4 & 19 & 21.1 & 87 & 85.6 \\
\hline \multicolumn{7}{|l|}{ Índice de masa corporal $\left(\mathrm{kg} / \mathrm{m}^{2}\right)$} \\
\hline • Normal (18.5-24.9) & 33 & 36.7 & 12 & 13.3 & 45 & 50.0 \\
\hline - Sobrepeso (25-29.9) & 26 & 28.9 & 7 & 7.8 & 33 & 36.7 \\
\hline - Obesidad clase I (30-34.9) & 6 & 6.7 & 6 & 6.7 & 12 & 13.3 \\
\hline \multicolumn{7}{|l|}{ Lipodistrofia } \\
\hline$\cdot \mathrm{Si}$ & 2 & 3.1 & 2 & 8.0 & 4 & 4.4 \\
\hline - No & 63 & 96.9 & 23 & 92.0 & 86 & 95.6 \\
\hline
\end{tabular}

\section{Discusión}

La distribución por género de esta población de pacientes VIH positivos difiere a los resultados obtenidos por otro estudio realizado por los mismos investigadores en una población VIH seropositiva del departamento del Atlántico, perteneciente a un régimen de salud subsidiado, es decir pacientes de nivel socioeconómico bajo (13). En dicho estudio el porcentaje de pacientes del sexo femenino fue mayor (46\%), lo que podría indicar un menor autocuidado o menor uso de medidas preventivas para las enfermedades de transmisión sexual en pacientes de menores recursos económicos.

Los datos de prevalencia de obesidad y sobrepeso de nuestro estudio son similares a los resultados obtenidos en la población colombiana en general hechos en la Encuesta Nacional de Salud (32\% sobrepeso, 14\% obesidad) (12).

Los resultados del perfil lipídico mostraron diferencias significativas en la elevación del nivel de triglicéridos entre quienes recibían tratamiento antirretroviral (inhibidores de proteasa) y los sin tratamiento, siendo este resultado consistente con datos publicados en las guías de manejo del VIH a nivel mundial (14). El nivel medio de colesterol de baja y alta densidad estuvo en límites normales. Al comparar estos hallazgos con una cohorte de 115 pacientes hipertensos de una seccional de EPS SURA de Barranquilla, con media de edad de 57 años, $16 \%$ de diabéticos y $65 \%$ mujeres, el nivel de triglicéridos de los pacientes VIH positivos fue mayor (197.8 mg/dL vs $171 \mathrm{mg} / \mathrm{dL}$ ) pero sus niveles de colesterol HDL y LDL fueron inferiores (15).

Revisando los datos de la investigación en pacientes VIH positivos realizada por Guevara y col (2008) en un hospital de tercer nivel de Bogotá, hallamos similitudes en lo referente a edad, distribución por sexo e IMC promedio (16). Sin embargo, se observan diferencias en la prevalencia de SM: $21 \%$ tanto en hombres como mujeres, frente a $37.8 \%$ en nuestro estudio, con $56 \%$ de prevalencia en mujeres 
Tabla 2. SM, Esquemas de tratamiento antirretroviral, perfil lipídico e IMC en pacientes con VIH.

\begin{tabular}{|c|c|c|c|}
\hline & Media & DE & Diferencia \\
\hline \multicolumn{4}{|l|}{ Triglicéridos en pacientes con SM } \\
\hline • TAG en pacientes con SM & 286.6 & 200.8 & $\mathrm{p}=0,003$ \\
\hline - TAG en pacientes sin SM & 144.3 & 79 & \\
\hline \multicolumn{4}{|l|}{$\begin{array}{l}\text { IMC en pacientes con y } \\
\sin \mathrm{SM} \text { y VIH }\end{array}$} \\
\hline - IMC en pacientes con SM & 27.6 & 3.4 & $\mathrm{p}=0,000$ \\
\hline - IMC en pacientes sin SM & 24.1 & 3.1 & \\
\hline \multicolumn{4}{|l|}{$\begin{array}{l}\text { CD4 Inicial en pacientes } \\
\text { VIH positivos }\end{array}$} \\
\hline - Pacientes con SM & 312.4 & 247.6 & $\mathrm{p}=0,003$ \\
\hline - Pacientes sin SM & 323.3 & 258.3 & \\
\hline \multicolumn{4}{|l|}{$\begin{array}{l}\text { Hipertrigliceridemia y tipo de } \\
\text { esquema de tratamiento ARV }\end{array}$} \\
\hline - Análogos no nucleósidos & 193.9 & 182 & $\mathrm{p}=0.1724^{*}$ \\
\hline - Inhibidores de proteasa & 226.5 & 121.6 & $\mathrm{p}=0.0188^{*}$ \\
\hline - Sin tratamiento & 145.7 & 76 & \\
\hline \multicolumn{4}{|l|}{$\begin{array}{l}\text { Colesterol HDL y tipo de esquema } \\
\text { de tratamiento ARV }\end{array}$} \\
\hline - Análogos no nucleósidos & 44.8 & 12.2 & $\mathrm{p}=0.1440 *$ \\
\hline - Inhibidores de proteasa & 41.6 & 14.1 & $\mathrm{p}=0.5260 *$ \\
\hline - Sin tratamiento & 39.2 & 8.7 & \\
\hline \multicolumn{4}{|l|}{$\begin{array}{l}\text { Colesterol LDL y tipo de esquema } \\
\text { de tratamiento ARV }\end{array}$} \\
\hline - Análogos no nucleósidos & 117.2 & 29 & $\mathrm{p}=0.5260^{*}$ \\
\hline - Inhibidores de proteasa & 102.2 & 30.1 & $\mathrm{p}=0.8575^{*}$ \\
\hline - Sin tratamiento & 104.1 & 29 & \\
\hline \multicolumn{4}{|l|}{$\begin{array}{l}\text { IMC y Tipo de esquema de } \\
\text { tratamiento ARV }\end{array}$} \\
\hline - Análogos no nucleósidos & 26.0 & 3.6 & $\mathrm{p}=0.2066^{*}$ \\
\hline - Inhibidores de proteasa & 24.3 & 3.5 & $\mathrm{p}=0.7064 *$ \\
\hline - Sin tratamiento & 24.7 & 2.7 & \\
\hline \multicolumn{4}{|l|}{$\begin{array}{l}\text { Tipo de esquema de tratamiento } \\
\text { ARV y SM }\end{array}$} \\
\hline - Análogos no nucleósidos (\% con SM) & 30.6 & & $\mathrm{p}=0.9905^{*}$ \\
\hline - Inhibidores de proteasa (\% con SM) & 46.4 & & $\mathrm{p}=0.8324 *$ \\
\hline - Sin tratamiento (\% con SM) & 36.4 & & \\
\hline \multicolumn{4}{|l|}{ Tipo de análogo nucleósido y SM } \\
\hline - Abacavir (\% con SM) & 41.9 & & $\mathrm{p}=0.0991 *$ \\
\hline -Zidovudina (\% con SM) & 22.2 & & $\mathrm{p}=0.6230^{*}$ \\
\hline - Sin tratamiento (\% con SM) & 36.4 & & \\
\hline \multicolumn{4}{|l|}{ Lipodistrofia (IMC) } \\
\hline - $\mathrm{Si}$ & 24.8 & 2.6 & $\mathrm{p}=0.6415$ \\
\hline$\cdot$ No & 25.5 & 3.7 & \\
\hline \multicolumn{4}{|l|}{ Lipodistrofia (índice de cintura) } \\
\hline - $\mathrm{Si}$ & 88.5 & 7.7 & $\mathrm{p}=0.4677$ \\
\hline$\cdot$ No & 91.8 & 9.3 & \\
\hline * Diferencias respecto a sin tratamiento & & & \\
\hline
\end{tabular}

La prevalencia de lipodistrofia, registrada como tal en la historia clínica de los pacientes fue de $4.4 \%$ en la totalidad de los pacientes y $5 \%$ en los pacientes recibiendo TARAE respectivamente. Estos datos son inferiores a los registrados en la revisión hecha por Waters y colaboradores en el año 2007, la cual fue de 14-40\%, en pacientes usando terapia antirretroviral (17). Esto podría deberse a la inclusión de nuevos medicamentos antirretrovirales que causen menos esta complicación.

Los hallazgos de esta investigación deben servir para implementar estrategias para prevenir el curso natural de la insulinorresistencia y en lo posible la aparición de la diabetes mellitus en pacientes VIH positivos. De acuerdo con los criterios de la Asociación Americana de Diabetes, el tamizaje para diabetes debería realizarse en pacientes mayores de 45 años cada tres años o con una frecuencia mayor en pacientes con IMC igual o superior a 25 , triglicéridos sobre $250 \mathrm{mg} / \mathrm{dL}$, HDL menor de $35 \mathrm{mg} / \mathrm{dL}$, hipertensión arterial, condiciones asociadas a insulinorresistencia, entre otros factores de riesgo (18), lo que aplica para muchos de nuestros pacientes VIH positivos.

Sugerimos realizar el algoritmo diagnóstico de la diabetes sugerido reciente por el Dr. Inzucci (2012), en el cual todo paciente con riesgo para diabetes, debe ser sometido a una glucosa en ayunas y un realización de hemoglobina glicosilada cada uno a tres años (18). De acuerdo con este algoritmo se podría clasificar a los pacientes en: pacientes con resultados normales, diabéticos, con glucosa en ayunas alterada y con hemoglobina glicosilada alterada; $y$, definir así su seguimiento y tratamiento, tanto farmacológico como no farmacológico. Estimamos además que como norma obligatoria todo programa para el manejo integral del VIH, una vez se establezca cuántos de sus pacientes se hallan con SM y/o intolerancia a los hidratos de carbono, se inicie un manejo intensivo y adecuado de la nutrición y el ejercicio de este grupo, donde se estimulen los cambios en el estilo de vida (CTEV). Se podría tomar como modelo la experiencia del estudio de prevención de diabetes (Diabetes Prevention Program) (19), el cual demostró una reducción del riesgo de desarrollar diabetes mellitus tipo 2 del $58 \%$, en el grupo de pacientes intolerantes a los carbohidratos con CTEV a los 2.8 años de seguimiento. En esta investigación la metformina fue relativamente equivalente al CTEV en pacientes jóvenes y con un índice de masa corporal superior a $35 \mathrm{~kg} / \mathrm{m}^{2}$. Cabe anotar que la intervención sobre el estilo de vida en este trabajo fue compleja y costosa, lo que hace poco repetible en el contexto de la práctica clínica habitual. Se requieren estudios sobre la prevención de la diabetes tipo 2 en los pacientes VIH positivos, para evaluar la relación costo efectividad de las medidas sugeridas en esta investigación.

\section{Agradecimientos}

Estudio financiado por SURA empresa promotora de salud.

Los autores declaramos no tener conflicto de interés con marca farmacéutica alguna. 


\section{Referencias}

1. Palella FJ, Jr., Delaney KM, Moorman AC, Loveless MO, Fuhrer J, Satten GA, et al. Declining morbidity and mortality among patients with advanced human immunodeficiency virus infection. HIV Outpatient Study Investigators. New Eng J Med 1998; 338(13): 853-60.

2. Barbaro G. Cardiovascular manifestations of HIV infection. J R Soc Med 2001; 94(8): 384-90.

3. Sabin CA, d'Arminio Monforte A, Friis-Moller N, Weber R, El-Sadr WM, Reiss $\mathbf{P}$, et al. Changes over time in risk factors for cardiovascular disease and use of lipid-lowering drugs in HIV-infected individuals and impact on myocardial infarction. Clinical infectious diseases : an official publication of the Infectious Diseases Society of America 2008; 46(7): 1101-10.

4. Friis-Moller N, Sabin CA, Weber R, d'Arminio Monforte A, El-Sadr WM, Reiss P, et al. Combination antiretroviral therapy and the risk of myocardial infarction. New Eng J Med 2003; 349(21): 1993-2003.

5. Bozzette SA, Ake CF, Tam HK, Chang SW, Louis TA. Cardiovascular and cerebrovascular events in patients treated for human immunodeficiency virus infection. New Eng J Med 2003; 348(8): 702-10.

6. Ho JE, Hsue PY. Cardiovascular manifestations of HIV infection. Heart 2009; 95(14): 1193-202.

7. Grinspoon SK, Grunfeld C, Kotler DP, Currier JS, Lundgren JD, Dube MP, et al. State of the science conference: Initiative to decrease cardiovascular risk and increase quality of care for patients living with HIV/AIDS: executive summary. Circulation 2008; 118(2): 198-210.

8. Lopez P. Tratamiento de la hipertensión arterial en el paciente con síndrome metabólico. Rev Colom Cardiol 2010; 17(1): 22-7.

9. Carr A, Samaras K, Burton S, Law M, Freund J, Chisholm DJ, et al. A syndrome of peripheral lipodystrophy, hyperlipidaemia and insulin resistance in patients receiving HIV protease inhibitors. AIDS 1998; 12(7): F51-8.

10. Carr A, Samaras K, Thorisdottir A, Kaufmann GR, Chisholm DJ, Cooper DA. Diagnosis, prediction, and natural course of HIV-1 protease-inhibitor-associated lipodystrophy, hyperlipidaemia, and diabetes mellitus: a cohort study. Lancet 1999; 353(9170): 2093-9.

11. Friis-Moller N, Thiebaut R, Reiss P, Weber R, Monforte AD, De Wit S, et al. Predicting the risk of cardiovascular disease in HIV-infected patients: the data collection on adverse effects of anti-HIV drugs study. European journal of cardiovascular prevention and rehabilitation: Official journal of the European Society of Cardiology, Working Groups on Epidemiology \& Prevention and Cardiac Rehabilitation and Exercise Physiology 2010; 17(5): 491-501.

12. Instituto Colombiano de Bienestar Familiar. Encuesta Nacional de la Situación Nutricional en Colombia, 2005. Primera edición ed. Bogotá: Panamericana Formas e Impresos S.A; 2006.

13. Polo P, Castañeda C, Sierra M, Alvis N. Prevalencia de Hepatitis B oculta en pacientes VIH positivos de una instituciòn de salud en Barranquilla Colombia. Infectio 2010; 14(1): $39-46$.

14. Panel on Antiretroviral Guidelines for Adults and Adolescents. Guidelines for the use of antiretroviral agents in HIV-1-infected adults and adolescents. Department of Health and Human Services. Available at http://www.aidsinfo.nih.gov/ contentfiles/lvguidelines/ adult and adolescentgl.pdf. .

15. Polo P, Manosalva D, Palacios D, Valverde C. Modificación del riesgo cardiovascular en pacientes asistentes al programa de prevención y promoción de hipertensión arterial en puesto de salud Las Palmas. Barranquilla, 2002-2004. XXII Congreso Colombiano de Cardiologia; Cartagena de Indias 2008.

16. Guevara F, Cañón B, Liévano M, Lombo B, Rendón I, Blanco F. Prevalence of metabolic syndrome in patients infected with HIV Using ATP III and IDF criteria. Acta Med Colomb 2008; 4(33 ): 282 - 8.

17. Waters L, Nelson M. Long-term complications of antiretroviral therapy: lipoatrophy. Int J Clin Pract 2007; 61(6): 999-1014.

18. Inzucchi SE. Clinical practice. Diagnosis of diabetes. New Eng J Med 2012; 367(6): 542-50.

19. Knowler WC, Barrett-Connor E, Fowler SE, Hamman RF, Lachin JM, Walker EA, et al. Reduction in the incidence of type 2 diabetes with lifestyle intervention or metformin. New Eng J Med 2002; 346(6): 393-403. 